\title{
Longitudinal growth curves for children with classical osteogenesis imperfecta (types III and IV) caused by structural pathogenic variants in type I collagen
}

\author{
Lauren A. Barber, MD ${ }^{1,3}$, Craig Abbott, $\mathrm{PhD}^{2}$, Vihang Nakhate, BA ${ }^{1,4}$, An N. Dang Do, MD, PhD ${ }^{2}$, \\ Angela R. Blissett, $\mathrm{PhD}^{1,5}$ and Joan C. Marini, MD, $\mathrm{PhD}^{1}$
}

Purpose: Growth deficiency is a cardinal feature of osteogenesis imperfecta (OI) types III and IV, caused by pathogenic variants in type I collagen. OI-specific longitudinal growth charts are needed for patient care.

Methods: We compiled longitudinal length, weight, head circumference, and body mass index (BMI) data from 100 children with types III and IV OI and known type I collagen pathogenic variants. Effects of gender, OI type, and pathogenic variant were examined using multilevel modeling. OI-specific centile curves were constructed using generalized additive model for location, scale, and shape (GAMLSS).

Results: OI type and gender, but not the specific mutated collagen gene, significantly affect stature, but only OI type affects weight. Head circumference was not significantly different by gender, type, or mutated gene. In both genders, length curves for types III and IV OI overlap and the type IV 95th centile curve overlaps the lower US
Centers for Disease Control and Prevention (CDC) curves for the general population. A pubertal growth spurt is generally absent or blunted in types III/IV OI. The body mass index 50th and 95th centile curves are distinctly shifted above respective US CDC curves in both genders.

Conclusions: OI type is a stronger contributing factor than gender for OI growth, while curves do not differ for COL1A1 versus COL1A2 pathogenic variants. Types III and IV OI-specific growth curves are presented.

Genetics in Medicine (2019) 21:1233-1239; https://doi.org/10.1038/s41436018-0307-y

Keywords: growth curves; osteogenesis imperfecta; type III OI; type IV OI

\section{INTRODUCTION}

Osteogenesis imperfecta (OI) is a well-known heritable disorder of connective tissue characterized by bone fragility and deformity. ${ }^{1}$ Most patients with OI have autosomal dominantly inherited pathogenic variants in the type I collagen genes, ${ }^{2}$ COL1A1 or COL1A2 $2^{2}$, resulting in structurally abnormal collagen (OI types II, III, IV; OMIM 166210, 259420 , and 166220, respectively) or a reduced amount of structurally normal collagen (OI type I, OMIM 166200). Nonlethal pathogenic variants causing types III and IV OI occur in both collagen chains, with a variety of substituting residues. $^{2}$

Significant short stature is a cardinal feature of types III and IV OI, the progressively deforming and moderately severe forms, respectively. Growth deficiency is generally marked by the end of the first year of life and continues to progress thereafter. ${ }^{3}$ Clinicians and families lack information necessary to monitor the growth of a child with OI. Difficulty in setting OI-specific growth expectations undermines detection of other medical conditions that involve growth failure.

Syndrome-specific growth charts are available for Down syndrome, ${ }^{4}$ Turner syndrome, ${ }^{5,6}$ and achondroplasia, ${ }^{7}$ but not for the most common forms of OI. The aims of this study were (1) to delineate growth differences between classical OI patients based on gender, OI type (III versus IV), and location of pathogenic variant (COL1A1 versus COL1A2); and (2) to construct standard length and weight growth curves, and derivative body mass index (BMI) curves for OI types III and IV.

\section{MATERIALS AND METHODS}

Growth parameters, length $(\mathrm{cm})$, weight $(\mathrm{kg})$, and head circumference $(\mathrm{cm})$, were measured at the National Institutes of Health (NIH) Clinical Center in 100 patients with OI types

\footnotetext{
${ }^{1}$ Section on Heritable Disorders of Bone and Extracellular Matrix, NICHD, NIH, Bethesda, MD, USA; ${ }^{2}$ Office of the Clinical Director, NICHD, NIH, Bethesda, MD, USA; ${ }^{3}$ Present address: Hospital for Special Surgery, New York, NY, USA; ${ }^{4}$ Present address: Harvard Medical School, Boston, MA, USA; ${ }^{5}$ Present address: The Ohio State University Wexner Medical Center, Columbus, OH, USA. Correspondence: Joan C. Marini (oidoc@helix.nih.gov)

These authors contributed equally: Lauren A. Barber, Craig Abbott and Vihang Nakhate
} 
III and IV caused by pathogenic variants in type I collagen. Patients were enrolled in the Eunice Kennedy Shriver National Institute of Child Health and Human Development (NICHD) longitudinal pediatric OI study from 1986 to 2006. Parameters were measured at intervals that depended on the frequency of patient visits, with median interval of 5.4 months (median minimal and maximal intervals were 2.8 months and 16 months, respectively). All measurements were obtained and documented by research nurses who are well-versed in these measurements, and by using the same equipment for all patients. Informed consent was obtained from parents for each participant in an NICHD institutional review board (IRB)-approved protocol on OI natural history.

\section{Demographics}

Similar to Centers for Disease Control and Prevention (CDC) pediatric growth charts, our OI growth charts cover the age range of 2-16 years. The distribution of gender, OI type, and collagen pathogenic variants are shown in Table S1, as are the total numbers of measurements. The study includes data from 1480 length, 1502 weight, and 1398 head circumference observations. For comparison curves between major parameters (gender, OI type, pathogenic variant location), 55 patients are female, while 45 are male. Forty-five patients have type III ( 27 females), while 55 patients have type IV OI (28 females). Pathogenic variants in COL1A1 are causative in 57 patients (30 type III OI), while COL1A2 pathogenic variants are causative in 43 patients (15 type III OI). Patients with null COL1A1 alleles were not included. Patients with scoliosis were included, because this is part of the natural history of OI contributing to short stature.

\section{Statistics}

Differences in growth curves based on gender, OI type, and location of pathogenic variant (COL1A1 versus COL1A2) were analyzed using nonlinear multilevel modeling. Analyses estimated the sample average growth curves across age (years) and tested for population group differences in size (intercept), growth rate (slope), and curvature (acceleration) of the growth curves. Details are included in Supplementary Materials and Methods.

\section{Standard growth curve construction}

Centile curves were constructed using the generalized additive model for location, scale, and shape (GAMLSS $)^{8}$ package from the R statistical computing environment. ${ }^{9}$ GAMLSS was adopted by the World Health Organization (WHO) in the development of its 2007 growth reference charts, and incorporates the LMS method ${ }^{10}$ of curve smoothing used by the CDC to develop its 2000 growth charts.

Data were binned into half-year intervals, with the lowest bin at 1.5 years and the highest bin at 17.5 years, to generate curves for ages 2-16 years. Age bins covered a range of \pm 0.25 years. Each patient was limited to one measurement per age bin per growth parameter. When patients had multiple assessments within the same half-year interval, the occasion that fell closest to the defined age bin or that provided the most complete information (i.e., had scores for the most growth parameters) was retained for analysis. Centile curves were constructed using the LMS method ${ }^{10}$ and the extension of LMS developed by Rigby and Stasinopoulos. Details are provided in the Supplementary Materials and Methods.

\section{RESULTS}

Ol type and gender, but not the specific mutated collagen chain, significantly influence length

For the comparison of length, weight, and head circumference between OI type, gender, and specific mutated collagen chain, we used a compilation of 100 individual longitudinal curves to generate projected trajectories of growth parameters (Fig. 1). Length differed most significantly by OI type and was also significantly different by gender (Fig. 1a). Males with OI are taller than females with $\mathrm{OI}$ at all ages (i.e., growth rate at 2 years old, $5.7 \mathrm{~cm} /$ year vs. $4.5 \mathrm{~cm} /$ year, $p<0.05$; at 8 years old, $4.7 \mathrm{~cm} /$ year vs. $3.7 \mathrm{~cm} /$ year, $p<0.05$; cf. Table S3a model 3 ), and the difference becomes larger with increasing age. Individuals with type IV OI are taller than those with type III OI at all ages (at 2, 8, and 16 years, in $\mathrm{cm}$ /year, 6.3 vs. $3.5, p<0.001 ; 5.3$ vs. $2.7, p<0.001 ; 4.0$ vs. $1.5, p<0.001$, respectively; cf. Table S3a model 2), and the difference increases with age. The composite curves for type IV are steeper than those for type III across the age span. However, we found no difference in length trajectory between OI caused by nonlethal pathogenic variants in COL1A2 versus COL1A1 (Fig. 1a).

We further analyzed length trajectories using within-group comparisons among each gender, OI type, or mutant collagen chain (Fig. 1b). Both males and females with type IV OI were taller than individuals with type III OI. Type IV boys have both taller and steeper curves than type IV girls. Type III boys and girls have similar growth as young children, but the slightly steeper type III male curve led to a significant difference in the late teen years. When length trajectories were analyzed by both mutant collagen chain and either gender or OI type, we again found no influence of the specific mutated collagen alpha chain.

\section{Weight differs significantly by OI type, but not gender or mutated collagen chain}

Weight trajectories do not differ significantly by gender but do differ by OI type. Type IV OI children, who are also taller than type III children, having significantly greater weight than those with type III throughout life (ages 2, 8, and 16 years: 10.1 vs. $8 \mathrm{~kg}, p<0.001 ; 21.5$ vs. $14.6 \mathrm{~kg}, p<0.001$; and 54.2 vs. $33.1 \mathrm{~kg}, p<0.001$, respectively), and with the difference in weight increasing with age due to a steeper type IV OI curve (Fig. 1c). The mutant chain had a minimal effect on weight trajectories at 8 years old $(20.5$ vs. $17.0 \mathrm{~kg}, p<0.05)$, but this distinction did not remain as children aged.

Head circumference of types III and IV OI does not differ by gender, type, or mutated collagen chain

Interestingly, the trajectories for growth of head circumference did not differ significantly by OI type, gender, or mutant collagen chain (Fig. 1d). 
a

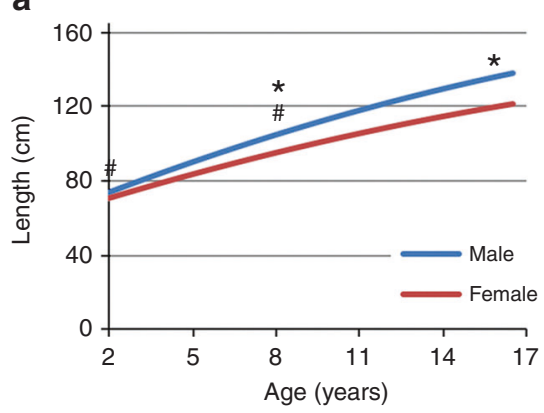

b

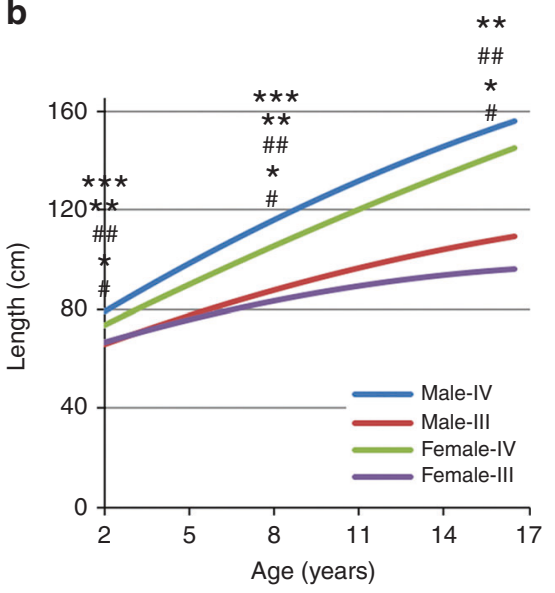

C
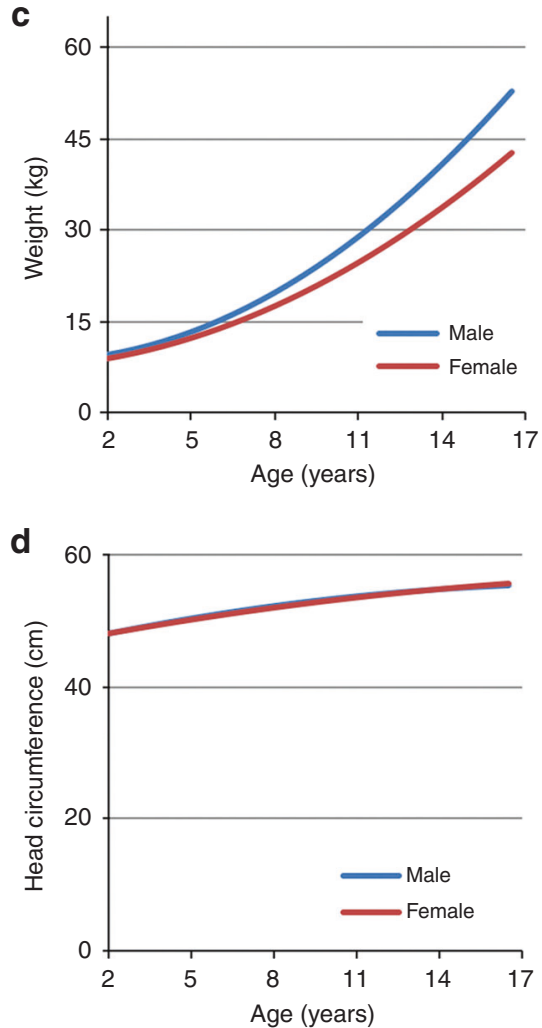
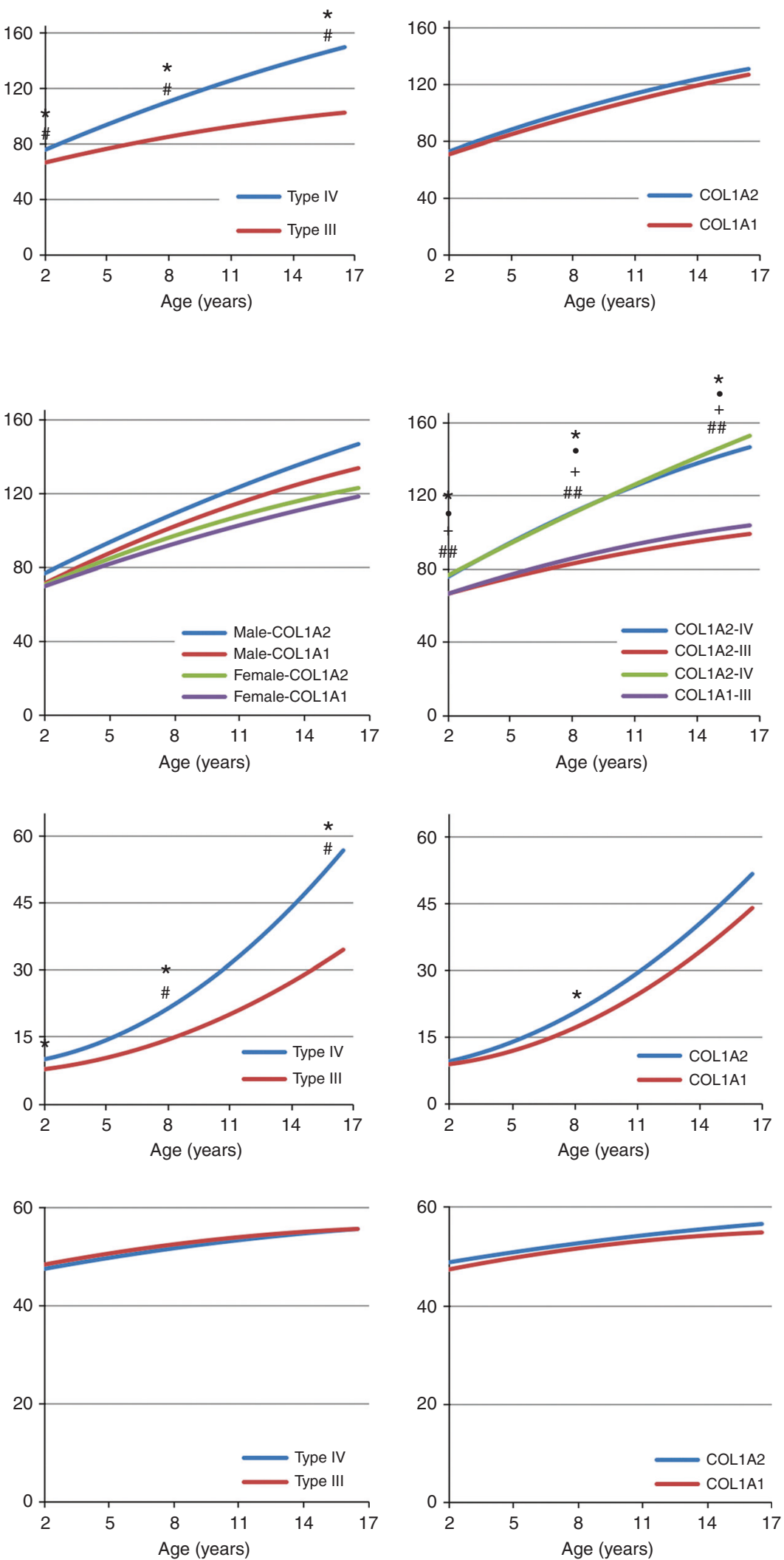

\section{Generation of standard OI curves}

To generate standard OI growth curves, length and weight data were binned into half-year intervals. Each patient was limited to 1 data point per bin so that patients with frequent visits did not skew the curves. Standardized OI growth charts are presented in Supplementary Figures S1-S11, by OI type 
Fig. 1 Comparison of composite longitudinal growth curves of children with types III and IV osteogenesis imperfecta (OI). (a) Comparison of longitudinal length curves by gender, OI type, and mutant collagen chain demonstrates that males are taller than females and children with type IV are taller than those with type III, while mutant type I collagen chain has no effect. (b) Comparison of longitudinal length curves by gender and OI type, gender and mutant collagen chain, and OI type and mutant collagen chain confirms the significant impact of OI type and lack of length correlation with specific mutant collagen chain. (c) Comparison of composite longitudinal weight curves by gender, Ol type, and mutant collagen chain reveals significant impact of Ol type. (d) Comparison of composite longitudinal curves of head circumference shows no impact of gender, Ol type, or specific mutant collagen chain. For $(\mathbf{a}, \mathbf{c}), *$ represents significant difference in length or weight, \# represents significant difference in rate of increase. For length compared by gender and OI type in (b), * is significant difference in length between type III and IV females, ** is significant difference in rate of length increase between types III and IV Ol females, \# is significant difference between male and female type IV OI, \#\# is significant difference in rate of length increase between types III and IV males, $* * *$ is significant difference in rate of length increase between male and female type IV OI children. For length compared by OI type and mutant chain in (b), * and + represent significant difference in length and growth rate, respectively, between types III and IV OI children with pathogenic variants in COL1A1; $\bullet$ and \#\# represents significant differences in length and growth rate, respectively, between types III and IV OI children with pathogenic variants in COL1A2. Significance noted at $p<0.05$.

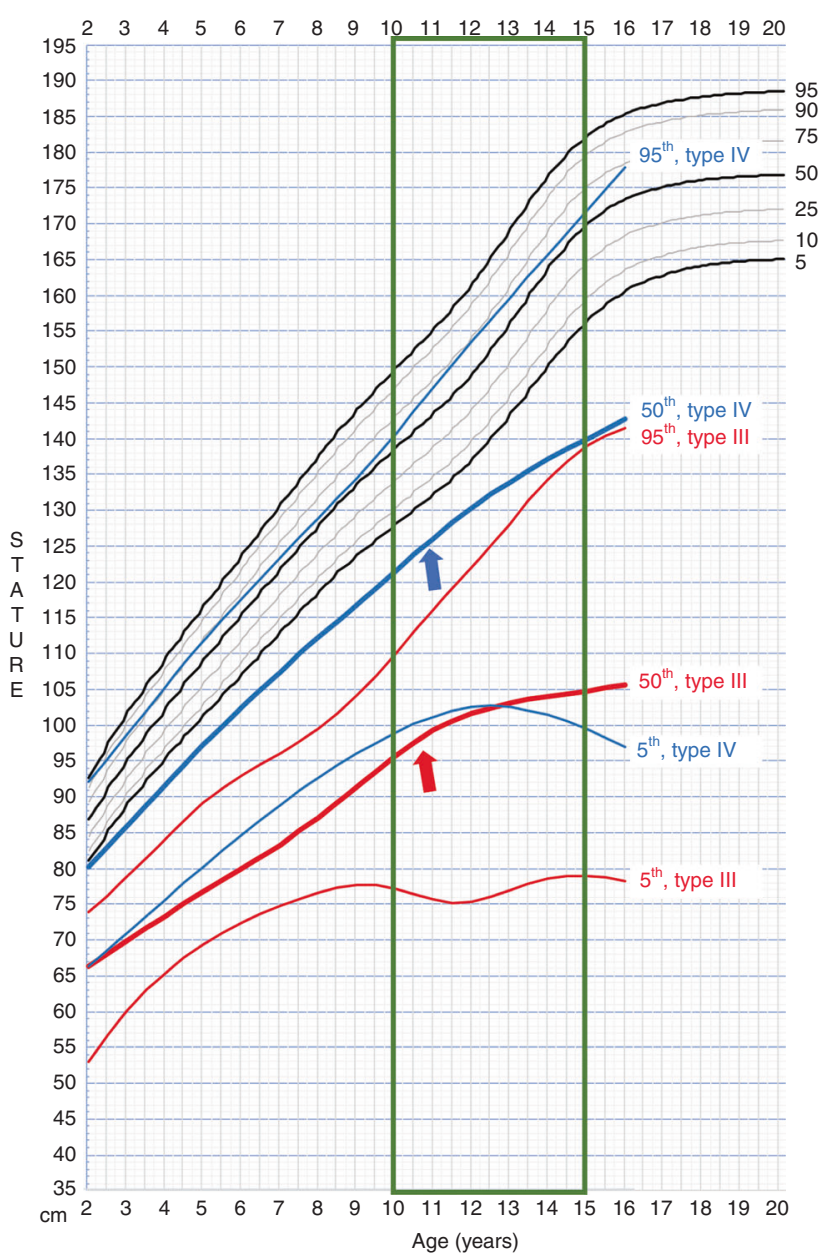

b

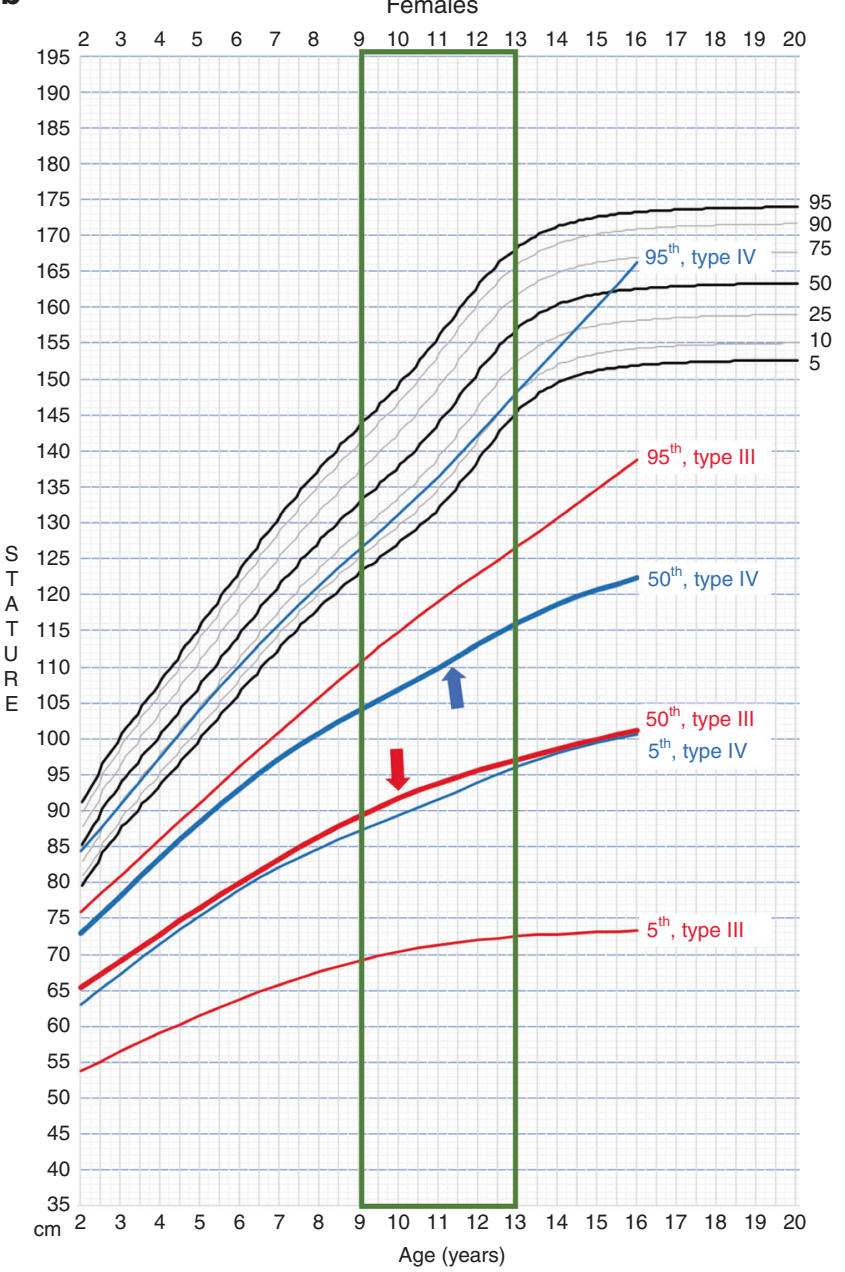

Fig. 2 Osteogenesis imperfecta (OI)-specific linear growth curves plotted against Centers for Disease Control and Prevention (CDC) growth curves for general US population. Growth charts for a males and $\mathbf{b}$ females. For both genders, the centile curves of types III (red) and IV (blue) overlap. The vertical box (green) highlights age range for pubertal growth spurt. Individuals with type III (red arrows) and IV (blue arrows) OI have blunted growth spurt.

and gender for length and weight. In addition, body mass index (BMI) values were calculated from length and weight data and the growth charts constructed for individual OI type and gender (Supplementary Figures S12-S16).

The median lengths of 2-year-old males and females with OI were $72.6 \mathrm{~cm}$ and $69.1 \mathrm{~cm}(z$-scores: -4.25 and -4.65 , respectively, versus the general population of children in the United States). The median lengths of 16-year-old males and females with OI were $124.4 \mathrm{~cm}$ and $112.8 \mathrm{~cm}(z$-score: -5.55 and -7.77 , respectively, versus US standard), respectively. The median weight of 2-year-old males and females were 8.54 and $8.13 \mathrm{~kg}$ ( $z$-score: -3.86 and -4.34 , respectively, versus US 
a

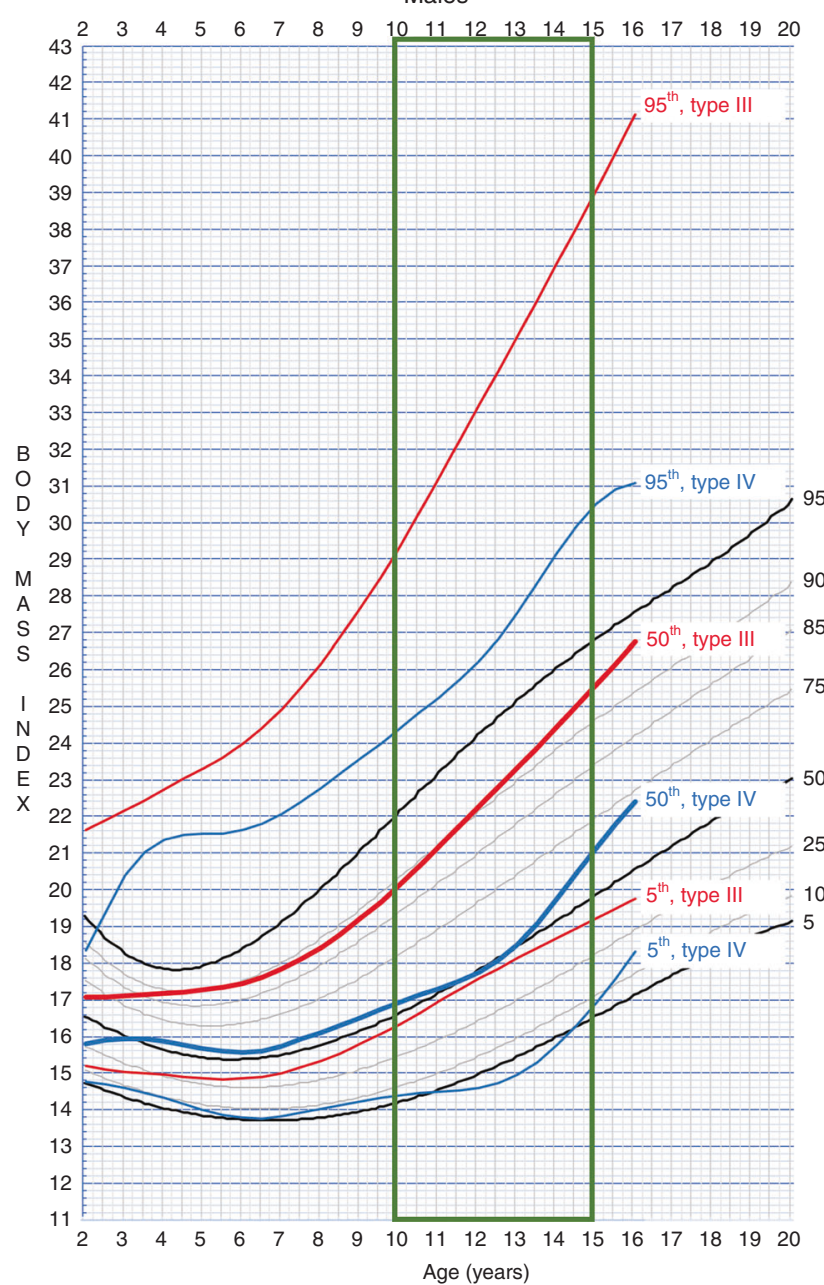

b

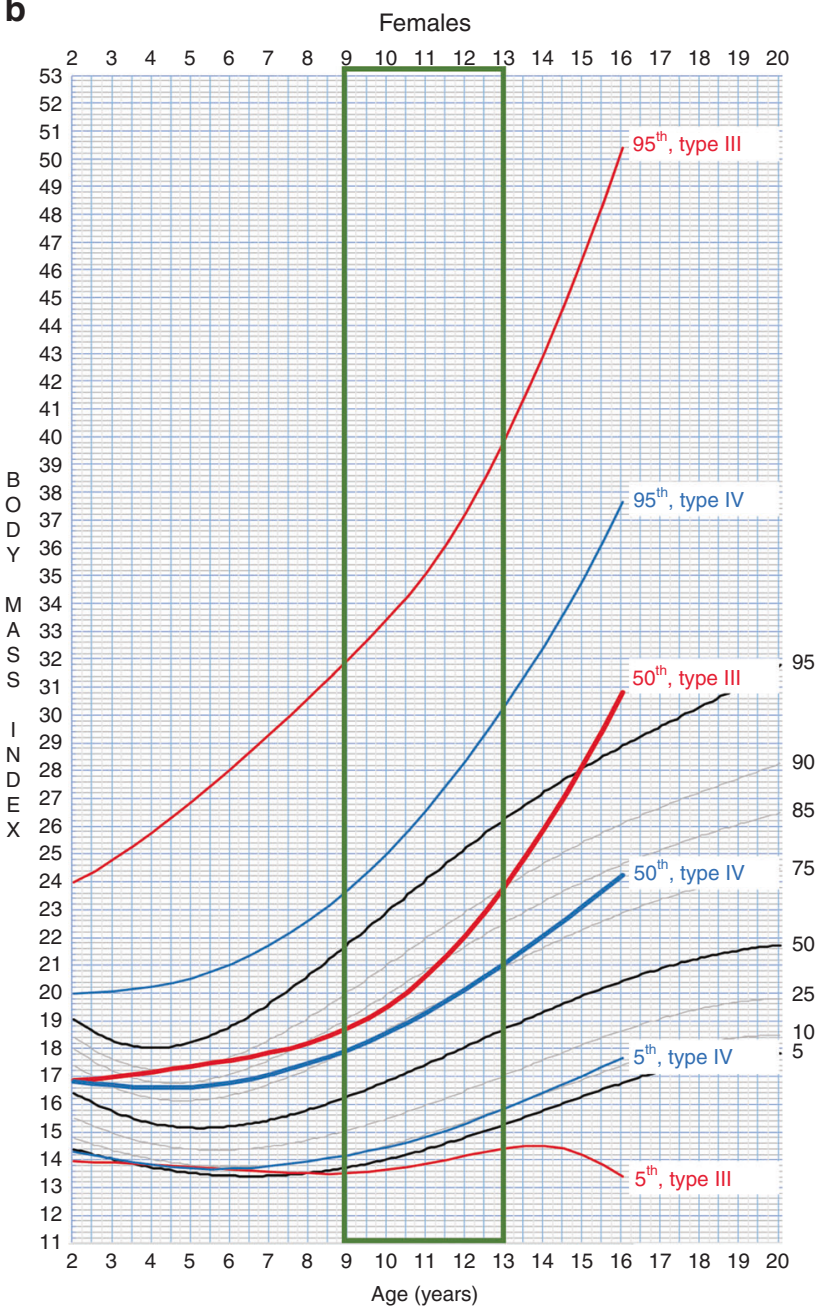

Fig. 3 Osteogenesis imperfecta (OI)-specific body mass index (BMI) growth curves plotted against Centers for Disease Control and Prevention (CDC) growth curves for general US population. Growth charts for (a) males and (b) females. Individuals with type III (red) have markedly higher BMI at all three centiles than those with type IV (blue) OI. The vertical box (green) highlights age range for pubertal growth spurt.

standard). At age 16 years, the mean weight for males and females was $33.4 \mathrm{~kg}(z$-score: -4.51$)$ and $35.3 \mathrm{~kg}(z$-score: $-3.65)$, respectively.

\section{Comparison of Ol-specific and general population length growth curves}

Direct comparison of OI-specific curves with the CDC length curves for the general US population ${ }^{11,12}$ are shown (Fig. 2). For both genders, there is overlap of the types III and IV OI centile curves. The type III OI 50th centile curve is similar to the type IV OI 5th centile. The tallest type III OI children (95th centile) are in the range of the average type IV OI children (50th centile). In both genders, the tallest type IV OI children (95th centile) have growth curves parallel to (females) or well into (males) the lower half of the general population curves. The OI length curves reflect a blunted pubertal growth spurt for both types as compared with the general population, with type III (red arrows) showing greater growth deceleration than type IV (blue arrows) OI individuals. ${ }^{13}$

\section{Comparison of BMI growth curves}

When plotted on the same scales and overlaid on CDC BMI curves, BMI curves in both genders for type III OI are markedly higher than corresponding type IV centile curves (Fig. 3). For example, type III OI 50th centile curve overlies the CDC 90th (Fig. 3a) and 85th-90th (Fig. 3b) centile curves; whereas, type IV OI 50th centile curve overlies the CDC 50th and 75th curves for males and females, respectively. This effect on BMI by OI type is most pronounced when considering the 95th centile growth curves in either gender.

Gender also contributes to differences in OI BMI growth curves. In males (Fig. 3a), type III curves for the 5th, 50th, and 95th centile all overlap with higher general population centile curves than those for type IV OI. The BMI slope of change in males with type III OI appears to be maintained from the pubertal period onward (Fig. 3a). The BMI slope of change in females continues to increase (Fig. $3 \mathbf{b}$ ), resulting in higher 50th and 95th centile curves for females than males after age 
12 years. For type IV OI, male BMI curves for the 50th and 95th centile track with the corresponding curves in the general population (Fig. 3a); whereas, female curves diverge upward after age 10 years starting with the 50th centile (Fig. 3b).

Overlapping of OI and the CDC general population BMI curves clearly demonstrates the presence of higher body mass index in a substantial proportion of individuals with OI (Fig. 3).

\section{DISCUSSION}

Significant growth deficiency is a cardinal feature of OI, but information on growth patterns in OI children has been sparse. The typical growth trajectories in types I, III, and IV $\mathrm{OI}^{3}$ are not suitable for comparison with individual patients. Lund et al. assembled the first cross-sectional data on length and weight of 86 patients with types I, III, and IV OI, including children and adults. ${ }^{14}$ They also separated patients into those with quantitative (OI type I) versus qualitative defects (OI types III and IV), although they did not distinguish between mutant collagen alpha chains. Type III/ IV OI patients were found to be shorter than type I OI patients. Head circumference was increased above the general population but did not differ significantly among OI types. North American cross-sectional data on OI growth were assembled from the initial treatment visit of 125 children in a pamidronate treatment trial. ${ }^{15}$ Types III and IV OI mean length curves were distinct and growth deficiency increased with severity of the dysplasia. Collagen pathogenic variantswere not analyzed. Most recently, cross-sectional data on 343 children and adults with $\mathrm{OI}^{16}$ also supported increasingly impaired growth with more severe OI types.

The study presented here compares longitudinal growth data of 100 children with types III and IV OI and known collagen pathogenic variants. Composite growth curves compare length, weight, and head circumference in types III and IV OI. Stature in OI is significantly affected by gender (males are taller) and OI type (type III individuals are shorter), but not by mutant collagen chain. Weight trajectories differ only by OI type, reflecting the greater size of type IV children. Head circumference does not differ by gender, type, or mutant collagen chain, in agreement with Lund. ${ }^{14}$ Although glycine substitutions are more likely to be lethal in COL1A1 than COL1A2 $2^{2}$, the nonlethal substitutions in our patients do not have a chain-specific effect on length, weight, or head circumference.

OI-specific length, weight, and BMI curves derived from the longitudinal data reveal substantial overlap in centiles between types III and IV OI. Individuals with either OI type have a blunted pubertal linear growth spurt. Individuals with type III OI have significantly higher BMI when compared with the general population.

The longitudinal linear growth curves presented here reflect preponderantly the intrinsic growth deficiency of the axial skeleton in OI. Other skeletal factors, such as bowing of long bones, vertebral compressions, and scoliosis, ${ }^{17}$ as well as popcorn formation at the metaphyses of long bones, ${ }^{18}$ also make contributions to growth deficiency in OI. Anecdotal observations in the NIH natural history cohort suggest a correlation between decreased growth rate and increased scoliosis during puberty. Growth deficiency in OI is not due to defects in endocrine aspects of the growth hormone $(\mathrm{GH})$ axis. ${ }^{19}$ The growth responsiveness of many type IV OI children to administration of exogenous recombinant growth hormone $(\mathrm{rGH})^{20}$ suggests end-organ resistance may be contributory. Additionally, the BMI growth curves effectively demonstrate the presence of significant obesity in the OI population. The relative contributions of decreased activity secondary to recurrent fractures and decreased mobility, and of intrinsic cellular factors, remain to be determined. These OI-specific growth curves will fill an ongoing need in clinical care of patients with OI.

\section{ELECTRONIC SUPPLEMENTARY MATERIAL}

The online version of this article (https://doi.org/10.1038/s41436018-0307-y) contains supplementary material, which is available to authorized users.

\section{ACKNOWLEDGEMENTS}

The authors thank the patients who participated in the NICHD Natural History of OI Protocol, the 9W and 1NW nurses for skillful measurements, and Aileen Barnes for assistance in manuscript assembly and submission. The data were collected under NICHD protocol 97-CH-0064, as approved by the NICHD IRB and funded by the NICHD IRP.

\section{DISCLOSURE}

The authors declare no conflicts of interest.

\section{REFERENCES}

1. Forlino A, Marini JC. Osteogenesis imperfecta. Lancet. 2016;387:1657-1671.

2. Marini JC, et al. Consortium for osteogenesis imperfecta mutations in the helical domain of type I collagen: regions rich in lethal mutations align with collagen binding sites for integrins and proteoglycans. Hum Mutat. 2007;28:209-221

3. Marini JC, Bordenick S, Heavner G, Rose S, Chrousos GP. Evaluation of growth hormone axis and responsiveness to growth stimulation of short children with osteogenesis imperfecta. Am J Med Genet. 1993; 45:261-264.

4. Myrelid A, Gustafsson J, Ollars B, Anneren G. Growth charts for Down's syndrome from birth to 18 years of age. Arch Dis Child. 2002;87:97-103.

5. Garcia Rudaz C, et al. Growth of Argentinian girls with Turner syndrome. Ann Hum Biol. 1995;22:533-544.

6. Lyon AJ, Preece MA, Grant DB. Growth curve for girls with Turner syndrome. Arch Dis Child. 1985;60:932-935.

7. Hoover-Fong JE, McGready J, Schulze KJ, Barnes H, Scott Cl. Weight for age charts for children with achondroplasia. Am J Med Genet A. 2007; 143A:2227-2235.

8. Rigby RA, Stasinopoulos DM. Generalized additive models for location, scale and shape. J R Stat Soc Ser C Appl Stat. 2005;54:507-554.

9. R Foundation for Statistical Computing. R: a language and environment for statistical computing. Vienna, Austria; 2017.

10. Cole TJ. Fitting smoothed centile curves to reference data. J R Stat Soc Ser A Stat Soc. 1988;151:385-418

11. Tanner JM, Davies PS. Clinical longitudinal standards for height and height velocity for North American children. J Pediatr. 1985; 107:317-329 
12. Hamill PV, Drizd TA, Johnson $C L$, Reed RB, Roche AF. NCHS growth curves for children birth-18 years. United States. Vital Health Stat 11. 1977;165:i-iv, 1-74.

13. Kelly $A$, et al. Age-based reference ranges for annual height velocity in US children. J Clin Endocrinol Metab. 2014;99:2104-2112.

14. Lund AM, Muller J, Skovby F. Anthropometry of patients with osteogenesis imperfecta. Arch Dis Child. 1999;80:524-528.

15. Zeitlin L, Rauch F, Plotkin H, Glorieux FH. Height and weight development during four years of therapy with cyclical intravenous pamidronate in children and adolescents with osteogenesis imperfecta types I, III, and IV. Pediatrics. 2003;111:1030-1036.

16. Germain-Lee EL, et al. Cross-sectional and longitudinal growth patterns in osteogenesis imperfecta: implications for clinical care. Pediatr Res. 2016;79:489-495.
17. Sato A, Ouellet J, Muneta T, Glorieux FH, Rauch F. Scoliosis in osteogenesis imperfecta caused by COL1A1/COL1A2 mutationsgenotype-phenotype correlations and effect of bisphosphonate treatment. Bone. 2016;86:53-57.

18. Obafemi AA, Bulas DI, Troendle J, Marini JC. Popcorn calcification in osteogenesis imperfecta: incidence, progression, and molecular correlation. Am J Med Genet A. 2008;146A:2725-2732.

19. Marini JC, et al. The growth hormone and somatomedin axis in short children with osteogenesis imperfecta. J Clin Endocrinol Metab. 1993; 76:251-256.

20. Marini JC, et al. Positive linear growth and bone responses to growth hormone treatment in children with types III and IV osteogenesis imperfecta: high predictive value of the carboxyterminal propeptide of type I procollagen. J Bone Mineral Res. 2003;18:237-243. 